\title{
Representations of Six-dimensional Mubarakazyanov Lie algebras
}

\section{G Thompson ${ }^{1 *}$, C Hettiarachchi ${ }^{2}, \mathrm{~N} \mathrm{Jones}^{3}$ and A Shabanskaya ${ }^{4}$}

${ }^{1}$ Department of Mathematics \& Statistics, The University of Toledo, 2801 W. Bancroft St., Toledo, OH 43606, USA

${ }^{2}$ Department of Mathematics, Northern Virginia Community College, USA

${ }^{3}$ Department of Mathematics \& Statistics, The University of Toledo, 2801 W. Bancroft St., Toledo, OH 43606, USA

${ }^{4}$ Department of Mathematics and Computer Science, Eastern Connecticut State University, 83 Windham Street, Willimantic, CT 06226, USA

\begin{abstract}
A corrected list of the Mubarakzyanov algebras, that is, six-dimensional indecomposable solvable Lie algebras for which the nilradical is five-dimensional, is presented. For each class of algebra a matrix representation and a system of vector fields representation is given. As a consequence it follows that the dimension of the minimal matrix representation of any six-dimensional Lie algebra is at most six.
\end{abstract}

Keywords: Lie algebra; Lie group; Mubarakzyanov algebra; Minimal matrix representation; Vector field representation MSC Classification: 17B99

\section{Introduction}

As long ago as 1963 Mubarazyanov classified real six-dimensional indecomposable solvable Lie algebras for which the nilradical is five-dimensional [1]. This paper is frequently referred to but suffers from a number of defects. Recently Shabanskaya and Thompson [2] reconsidered Mubarazyanov's paper and made a number of improvements. Many errors, both substantive and typographical were corrected, some algebras were shown to be redundant, others missing; finally even in those algebras that were essentially correct, in many classes of algebras that depend on parameters, the parameters could be simplified and some removed entirely.

In the present article, given that Mubarazyanov's list has now been corrected, we turn to the issue of finding representations. We know of course that finding representations for solvable algebras is a completely different enterprise from the semi-simple case. In most cases the algebra will have a trivial center and so the adjoint representation furnishes a faithful representation. However, using the adjoint representation is not always satisfactory. In the first place many algebras have non-trivial centers; secondly, it frequently happens that in a class of algebras that depend on parameters the center will be non-trivial except for certain special values of the parameters. Therefore, one has to use a different representation in the exceptional case, which is an unsatisfactory state of affairs. In the representations given below we have endeavored to the extent possible to avoid such split cases.

We give two kinds of representations for the solvable algebras. First of all we consider matrix representations, in most cases as subalgebras of $\mathrm{gl}(6, \mathrm{R})$ or occasionally $\mathrm{gl}(5, \mathrm{R})$. However, there is something of a logistical problem in that it is wasteful of space to present a list of six matrices for each class of algebras. Alternatively, one could write a single matrix, $M$ say, depending on some variables such that the span of the matrices engenders the given algebra. In this paper we go one step further and simply convert the given $5 \times 5$ or $6 \times 6$ into a single 25 or 36 row-vector, that we call the DNA vector, by using the rows of $M$.

In the second kind of representation we give a system of six vector fields on $\mathrm{R}^{6}$ using local coordinates $(p, q, x, y, z, w)$, which furnish a basis for the algebra under Lie bracket. Actually, in our opinion the best way to represent a solvable algebra is to give the matrix Lie group that is engendered by exponentiating the space of matrices spanned by the matrix $M$ referred to in the preceding paragraph. It is then a simple matter to construct the matrix Lie algebra by differentiating and evaluating at the identity. One can also easily construct a system of left or right-invariant vector fields starting from the group matrix. All of these quantities can be obtained in principle from the DNA-vector, which explains the name. We hope eventually to publish such a list of matrices in a suitable venue but again space is an issue.

There is another interesting consequence of our list of matrix representations. Following Burde [3,4] we define the invariant $\mu$ $(g)$ to be the minimum value of $p$ such that a Lie algebra ${ }_{p \in I}$ has a representation as a subalgebra of $g l(p, R)$. Then we can assert that $\mu \leq 6$ for all of the Mubarakzyanov algebras. Ado's Theorem states that there exists a finite-dimensional faithful representation as a subalgebra of $g l$ $(p, R)$ for some $p \in N$. The theorem does not give much information about the value of $p$ but leads one to believe that $p$ may be very large in relation to the size of $n$ where $n$ is the dimension of $g$. A little care must be exercised because there may well be inequivalent representations for which this minimum value is attained. Of course if $g$ has a trivial center then the adjoint representation furnishes a faithful representation of $g$ and in the notation used above. $\mu \leq n$. Nonetheless many algebras have non-trivial centers, nilpotent algebras for example, and then the adjoint representation is not faithful. Even if the center is trivial it could well be the case that $\mu(g)<n$.

It has been shown in Ghanam et al. [5] that for all five-dimensional algebras $\mu(g) \leq 5$. If we refer Ghanam et al. and Rawashdeh and Thompson [6,7] then we can assert quite generally that for all sixdimensional algebras $\mu(g) \leq 6$.

Of course it is interesting to ascertain the value of $\mu$ from a theoretical point of view. However, an important practical reason is that calculations involving symbolic programs such as Maple and Mathematica use up lots of memory when storing matrices; accordingly, calculations are likely to be faster if one can represent matrix Lie algebras using matrices

*Corresponding author: G Thompson, Department of Mathematics and Statistics, The University of Toledo, 2801 W. Bancroft St., Toledo, OH 43606, USA, Tel: + 734837 9283; E-mail: GERARD.THOMPSON@UTOLEDO.EDU

Received September 05, 2013; Accepted May 02, 2014; Published May 10, 2014

Citation: Thompson G, Hettiarachchi C, Jones N, Shabanskaya A (2014) Representations of Six-dimensional Mubarakazyanov Lie algebras. J Generalized Lie Theory Appl 8: 211. doi: 10.4172/1736-4337.1000211

Copyright: $\odot 2014$ Thompson G. This is an open-access article distributed under the terms of the Creative Commons Attribution License, which permits unrestricted use, distribution, and reproduction in any medium, provided the original author and source are credited. 
Citation: Thompson G, Hettiarachchi C, Jones N, Shabanskaya A (2014) Representations of Six-dimensional Mubarakazyanov Lie algebras. J Generalized Lie Theory Appl 8: 211. doi: 10.4172/1736-4337.1000211

Page 2 of 10

of a small size. In this paper the many calculations were performed with the help of Maple.

This article is organized as follows. In Table 2, we discuss the classification of the low-dimensional Lie Algebras. In Table 3, we list all the possible five-dimensional nilpotent algebras that occur as nilradicals in the Mubarakzyanov algebras. In Table 4, we reproduce mutatis mutandis the updated list of Mubarakzyanov algebras that appeared in Shabanskaya and Thompson [2]. In Table 5, we provide matrix representations for all the Mubarakzyanov algebras using the device of DNA-vectors introduced above. Finally in Table 6, we supply the list of vector field representations. In these last two Sections in the interests of efficiency we are content simply to provide the lists without any accompanying theory.

The second author acknowledges the support of the University of Toledo in the form of a summer research undergraduate fellowship.

\section{Classification of Low-Dimensional Lie Algebras}

Many authors have considered the classification problem for the low-dimensional Lie algebras. The classification of Lie algebras in two and three dimensions has been known for almost a century and dimensions four, five and six were carried out by two Russian mathematicians Morozov and Mubarakzyanov amongst others $[1,8,9]$. For a much more recent account the reader may also consult Basarab et al. and Campoamor $[10,11]$.

An important inequality for a solvable Lie algebra $g$ asserts that

$$
\operatorname{dim}(\mathfrak{n i l}(\mathfrak{g})) \geq \frac{1}{2} \operatorname{dim}(\mathfrak{g})
$$

where $\operatorname{nil}(g)$ denotes the nilradical of $g$ [8]. In dimension six there are four possible values for $\operatorname{dim}(\operatorname{nil}(g))$, that is; three, four, five or six. Case three does not occur because such a Lie algebra is decomposable. Case six comprises six-dimensional nilpotent Lie algebras that were classified by Morozov [8] and in the latter reference representations were supplied [12]. As regards the classification of the five-dimensional nilradical algebras they were studied by Mubarakzyanov [1], who found ninety nine classes of such Lie algebra; his list has been updated in Shabanskaya and Thompson [2] and this class of algebras is the concern of the present article. In the last case, that is the dimension of the nilradical is four, the algebras were classified by Turkowski [13]. A preliminary list of representations for the Turkowski algebras has been carried out by Rawashdeh and Thompson [7]. More details concerning the classification of the low-dimensional Lie algebras may be found in Turkowski [14].

\section{The Nilradical}

The nilradical nil $(g)$ of an indecomposable six-dimensional solvable Lie algebra $\mathfrak{g}$ for which $\mathfrak{n i l}(\mathfrak{g})$ is five-dimensional can be one of nine types. In his paper Mubarazyanov devotes a separate paragraph corresponding to each of these nine possible nilradicals.

- algebras 1 to 12 have abelian nilradical $\mathrm{R}^{5}$ (Table 1 )

- algebras 13 to 38 have nilradical isomorphic to $\mathbb{R}^{2} \oplus H$ (Table 2)

- algebras 39 to 53 have nilradical isomorphic to $\mathbb{R} \oplus A_{4,1}$ (Table 3 )

- algebras 54 to 70 have nilradical isomorphic to $A_{5,1}$ (Table 4)

- algebras 71 to 75 have nilradical isomorphic to $A_{5,2}$ (Table 5)

- algebras 76 to 81 have nilradical isomorphic to $A_{5,3}$ (Table 6)

- algebras 82 to 93 have nilradical isomorphic to $A_{5,4}$ (Table 7)

- algebras 94 to 98 have nilradical isomorphic to $A_{5,5}$ (Table 8)

- algebra 99 has nilradical isomorphic to $A_{5,6}$ (Table 9).

Here $\mathrm{R}^{\mathrm{n}}$ denotes the $n$-dimensional abelian Lie algebra, $H$ denotes the three-dimensional Heisenberg algebra, $A_{4,1}$ and the algebras $A_{5, n}$ are taken from [12] and denote nilpotent algebras, 4 and 5, respectively, being the dimensions of the algebra.

\section{Classification of the Mubarakzyanov Algebras}

In this Section we give an amended version of Mubarakzyanov's list of algebras. We have simplified the notation using for the parameters $a, b, c, d$. Also $\varepsilon$ can only assume the values of 0 or \pm 1 and $\delta$ can only be 0 or 1 and $\alpha$ can only be 0 or 2 except in $g_{6,8,8}$. We do not consider $\delta$ and $\varepsilon$ to be parameters since they only assume discrete values. At the start of each table we supply the brackets for the nilradical and in the table the brackets of the basis vectors with $e_{6}$. We do not highlight algebras where we merely improve the range of values of the parameters.

- Algebras below that have no asterisks are considered to be essentially correct and differ from Mubarakzyanov's algebras mutatis mudandis, that is, only by small notational differences

- Algebras that have one asterisk denote algebras in which the parameters in Mubarakzyanov's algebra can be simplified or an entry can be removed completely

\begin{tabular}{|c|c|c|c|c|c|c|}
\hline Algebra & {$\left[\mathrm{e}_{1}, \mathrm{e}_{6}\right]$} & {$\left[\mathrm{e}_{2}, \mathrm{e}_{6}\right]$} & {$\left[\mathrm{e}_{3}, \mathrm{e}_{6}\right]$} & {$\left[\mathrm{e}_{4}, \mathrm{e}_{6}\right]$} & {$\left[\mathrm{e}_{5}, \mathrm{e}_{6}\right]$} & Conditions on parameters \\
\hline $\operatorname{tg}_{6,1}$ & $\mathrm{e}_{1}$ & $\mathrm{ae}_{2}$ & $\mathrm{be}_{3}$ & $\mathrm{ce}_{4}$ & $\mathrm{de}_{5}$ & $0<|\mathrm{d}| \leq|\mathrm{c}| \leq|\mathrm{b}| \leq|\mathrm{a}| \leq 1$ \\
\hline $\operatorname{tg}_{6,2}$ & $\mathrm{ae}_{1}$ & $\mathrm{e}_{1}+\mathrm{ae}_{2}$ & $\mathrm{e}_{3}$ & $\mathrm{be}_{4}$ & $\mathrm{ce}_{5}$ & $0<|c| \leq|b| \leq 1$ \\
\hline $\operatorname{tg} 6,3$ & $\mathrm{ae}_{1}$ & $\mathrm{e}_{1}+\mathrm{ae}_{2}$ & $\mathrm{e}_{2}+\mathrm{ae}_{3}$ & $\mathrm{e}_{4}$ & $\mathrm{be}_{5}$ & $0<|b| \leq 1$ \\
\hline $\operatorname{tg} 6,4$ & $\mathrm{ae}_{1}$ & $\mathrm{e}_{1}+\mathrm{ae}_{2}$ & $\mathrm{e}_{2}+\mathrm{ae}_{3}$ & $e_{3}+a e_{4}$ & $\mathrm{e}_{5}$ & \\
\hline$g_{6,5}$ & $e_{1}$ & $\mathrm{e}_{1}+\mathrm{e}_{2}$ & $\mathrm{e}_{2}+\mathrm{e}_{3}$ & $e_{3}+e_{4}$ & $e_{4}+e_{5}$ & \\
\hline $\operatorname{tg}_{6,6}$ & $\mathrm{e}_{1}$ & $\mathrm{ae}_{2}$ & $\mathrm{e}_{2}+\mathrm{ae}_{3}$ & $\mathrm{be}_{4}$ & $e_{4}+b e_{5}$ & $a \leq b$ \\
\hline $\mathrm{g}_{6,7}$ & $\mathrm{ae}_{1}$ & $\mathrm{e}_{1}+\mathrm{ae}_{2}$ & $e_{2}+a e_{3}$ & $\mathrm{be}_{4}$ & $e_{4}+b e_{5}$ & $a=0, b=1$ or $a=1$ \\
\hline $\operatorname{tg}_{6,8}$ & $\mathrm{ae}_{1}$ & $\mathrm{be}_{2}$ & $\mathrm{ce}_{3}$ & $\mathrm{de}_{4}+\mathrm{e}_{5}$ & $-e_{4}+d e_{5}$ & $0<|\mathrm{c}| \leq|\mathrm{b}| \leq|\mathrm{a}|$ \\
\hline $\operatorname{tg}_{6,9}$ & $\mathrm{ae}_{1}$ & $\mathrm{be}_{2}$ & $\mathrm{e}_{2}+\mathrm{be}_{3}$ & $\mathrm{ce}_{4}-\mathrm{e}_{5}$ & $\mathrm{e}_{4}+\mathrm{ce}_{5}$ & $a \neq 0$ \\
\hline$g_{6,10}$ & $\mathrm{ae}_{1}$ & $\mathrm{e}_{1}+\mathrm{ae}_{2}$ & $\mathrm{e}_{2}+\mathrm{ae}_{3}$ & $\mathrm{be}_{4}-\mathrm{e}_{5}$ & $e_{4}+b e_{5}$ & \\
\hline $\operatorname{tg}_{6,11}$ & $e_{1}$ & $a e_{2}-b_{3}$ & $\mathrm{be}_{2}+\mathrm{ae}_{3}$ & $\mathrm{ce}_{4}-\mathrm{de}_{5}$ & $\mathrm{de}_{4}+\mathrm{ce}_{5}$ & $b d \neq 0$ \\
\hline $\operatorname{tg}_{6,12}$ & $\mathrm{ae}_{1}$ & $\mathrm{be}_{2}-\mathrm{e}_{3}$ & $e_{2}+b e_{3}$ & $e_{2}+b_{4}-e_{5}$ & $e_{3}+e_{4}+b e_{5}$ & $a \neq 0$ \\
\hline
\end{tabular}

Table 1: Lie algebras having nilradical isomorphic to $R^{5}$. 
Citation: Thompson G, Hettiarachchi C, Jones N, Shabanskaya A (2014) Representations of Six-dimensional Mubarakazyanov Lie algebras. J Generalized Lie Theory Appl 8: 211. doi: 10.4172/1736-4337.1000211

Page 3 of 10

\begin{tabular}{|c|c|c|c|c|c|c|}
\hline Algebra & {$\left[\mathrm{e}_{1}, \mathrm{e}_{6}\right]$} & {$\left[\mathrm{e}_{2}, \mathrm{e}_{6}\right]$} & {$\left[\mathrm{e}_{3}, \mathrm{e}_{6}\right]$} & {$\left[\mathrm{e}_{4}, \mathrm{e}_{6}\right]$} & {$\left[\mathrm{e}_{5}, \mathrm{e}_{6}\right]$} & Remarks \\
\hline$g_{6,13}$ & $(a+b) e_{1}$ & $\mathrm{ae}_{2}$ & $\mathrm{be}_{3}$ & $\mathrm{e}_{4}$ & $\mathrm{ce}_{5}$ & $a 2+b 2 \neq 0,|a| \leq|b|, 0<|c| \leq 1$ \\
\hline$g_{6,14}$ & $(a+b) e_{1}$ & $\mathrm{ae}_{2}$ & $\mathrm{be}_{3}$ & $\mathrm{e}_{4}$ & $e_{1}+(a+b) e_{5}$ & $|\mathrm{a}| \leq|\mathrm{b}|$ \\
\hline$g_{6,15}$ & $(a+1) e_{1}$ & $\mathrm{e}_{2}+\mathrm{e}_{4}$ & $\mathrm{ae}_{3}+\mathrm{e}_{5}$ & $\mathrm{e}_{4}$ & $\mathrm{ae}_{5}$ & $|a| \leq 1$ \\
\hline$g_{6,16}$ & $\mathrm{e}_{1}$ & $\mathrm{e}_{2}+\mathrm{e} 4$ & $\mathrm{e}_{5}$ & $e_{1}+e_{4}$ & 0 & \\
\hline $\operatorname{tg}_{6,17.1}$ & $\mathrm{ae}_{1}$ & $\mathrm{ae}_{2}$ & e4 & $\delta e_{1}$ & $\mathrm{e}_{5}$ & $(\delta=0,1)$ together $\mathrm{g} 6,17$ in $[1])$ \\
\hline$g_{6,17.2}{ }^{* * *}$ & $\mathrm{e}_{1}$ & $\mathrm{e}_{2}$ & $\mathrm{e}_{4}$ & 0 & $e_{1}+e_{5}$ & \\
\hline $\operatorname{tg}_{6,18}$ & $(a+1) e_{1}$ & $\mathrm{ae}_{2}$ & $\mathrm{e}_{3}+\mathrm{e}_{4}$ & $\mathrm{e}_{4}$ & $\mathrm{be}_{5}$ & $b \neq 0$ \\
\hline$g_{6,19}$ & $(a+1) e_{1}$ & $\mathrm{ae}_{2}$ & $e_{3}+e_{4}$ & $\mathrm{e}_{4}$ & $e_{1}+(a+1) e_{5}$ & \\
\hline $\operatorname{tg}_{6,20}$ & $\mathrm{e}_{1}$ & 0 & $\mathrm{e}_{3}+\mathrm{e}_{4}$ & $e_{1}+e_{4}$ & $\mathrm{be}_{5}$ & $b \neq 0$ \\
\hline $\operatorname{tg}_{6,21}$ & $2 \mathrm{ae}_{1}$ & $\mathrm{ae} 2+\mathrm{e} 3$ & $\mathrm{ae}_{3}$ & $\mathrm{e}_{4}$ & $\mathrm{be}_{5}$ & $0<|b| \leq 1$ \\
\hline $\mathrm{g}_{6,22}$ & $2 \mathrm{ae}_{1}$ & $\mathrm{ae} 2+\mathrm{e} 3$ & $\mathrm{ae}_{3}$ & $\mathrm{e}_{4}$ & $e_{1}+2 a e_{5}$ & \\
\hline $\mathrm{g}_{6,23}^{*}$ & $2 \delta e_{1}$ & $\delta e_{2}+e_{3}$ & $\delta e_{3}+e_{4}$ & $\delta e_{4}$ & $\epsilon \mathrm{e}_{1}+(2 \delta+a) \mathrm{e}_{5}$ & $\begin{array}{c}(\delta, \epsilon, a)=(1,0, \neq-2) \text { or }(0,0,1)(6.23 .1) \\
\text { (suspensions) or }(1,1,0)(6.23 .2)\end{array}$ \\
\hline $\operatorname{tg}_{6,24}{ }^{*}$ & 0 & $\mathrm{e}_{3}$ & $\mathrm{e}_{4}$ & $\mathrm{e}_{1}$ & $\mathrm{e}_{5}$ & \\
\hline$g_{6,25}$ & $(a+1) e_{1}$ & $\mathrm{e}_{2}$ & $\mathrm{ae}_{3}$ & $\mathrm{be}_{4}+\mathrm{e}_{5}$ & $\mathrm{be}_{5}$ & $|a| \leq 1$ \\
\hline$g_{6,26}$ & $(a+1) e_{1}$ & $\mathrm{e}_{2}$ & $\mathrm{ae}_{3}$ & $(a+1) e_{4}+e_{5}$ & $e_{1}+(a+1) e_{5}$ & $|a| \leq 1$ \\
\hline $\mathrm{g}_{6,27}{ }^{*}$ & $(a+b) e_{1}$ & $\mathrm{ae}_{2}$ & $\mathrm{be}_{3}+\mathrm{e}_{4}$ & $\mathrm{be}_{4}+\mathrm{e}_{5}$ & $\delta e_{1}+b e_{5}$ & $a=1, \delta=0$ or $a=0, b=1, \delta=0,1$ \\
\hline$g_{6,28}$ & $2 e_{1}$ & $\mathrm{e}_{2}+\mathrm{e} 3$ & $\mathrm{e}_{3}$ & $\mathrm{be}_{4}+\mathrm{e}_{5}$ & $\mathrm{be}_{5}$ & \\
\hline$g_{6,29}$ & $2 \mathrm{e}_{1}$ & $\mathrm{e}_{2}+\mathrm{e}_{3}$ & $\mathrm{e}_{3}$ & $2 \mathrm{e}_{4}+\mathrm{e}_{5}$ & $e_{1}+2 e_{5}$ & \\
\hline$g_{6,30}$ & 0 & $\mathrm{e}_{3}$ & 0 & $e_{4}+e_{5}$ & $\mathrm{e}_{5}$ & \\
\hline$g_{6,31}$ & $2 \mathrm{e}_{1}$ & $\mathrm{e}_{2}+\mathrm{e} 3$ & $\mathrm{e}_{3}+\mathrm{e} 4$ & $e_{4}+e_{5}$ & $\mathrm{e}_{5}$ & \\
\hline $\mathrm{g}_{6,32}^{* * * *}$ & $2 \mathrm{ae}_{1}$ & $a e_{2}+e_{3}$ & $\mathrm{ae}_{3}-\mathrm{e}_{2}$ & $\begin{array}{c}\delta e_{1}+(2 a+ \\
\text { b) } e_{4}\end{array}$ & $\mathrm{ce}_{5}$ & $(\delta, b)=(0, \neq-2 a)$ or $=(1,0), c \neq 0,2 a+b \geq c$ \\
\hline $\operatorname{tg}_{6,32}^{\prime}$ & $2 \mathrm{ae}_{1}$ & $\mathrm{ae}_{2}+\mathrm{e}_{3}$ & $\mathrm{ae}_{3}-\mathrm{e}_{2}$ & $\mathrm{be}_{4}$ & $\mathrm{ce}_{5}$ & $a \geq 0,0<|b| \leq|c|$ \\
\hline$g_{6,33}^{* * * *}$ & $2 \mathrm{ae}_{1}$ & $\mathrm{ae}_{2}+\mathrm{e} 3$ & $\mathrm{ae}_{3}-\mathrm{e}_{2}$ & $\mathrm{be}_{4}$ & $e_{1}+2 a_{5}$ & $b \neq 0, b \leq 2 a$ \\
\hline $\operatorname{tg}_{6,33}^{\prime}$ & $2 \mathrm{ae}_{1}$ & $\mathrm{ae}_{2}+\mathrm{e} 3$ & $\mathrm{ae}_{3}-\mathrm{e}_{2}$ & $\mathrm{e} 1+2 \mathrm{ae}_{4}$ & $\mathrm{be}_{5}$ & $a \geq 0, b \neq 0$ \\
\hline $\mathrm{g}_{6,34}{ }^{*}$ & $2 \mathrm{ae}_{1}$ & $\mathrm{ae}_{2}+\mathrm{e}_{3}$ & $\mathrm{ae}_{3}-\mathrm{e}_{2}$ & $(2 a+b) e_{4}+e_{5}$ & $\delta e_{1}+(2 a+b) e_{5}$ & $a \geq 0, \delta=0$ or $\delta=1, b=0$ \\
\hline$g_{6,35}$ & $(a+b) e_{1}$ & $\mathrm{ae}_{2}$ & $\mathrm{be}_{3}$ & $\mathrm{ce}_{4}+\mathrm{e}_{5}$ & $\mathrm{Ce}_{5}-\mathrm{e}_{4}$ & $a 2+b 2 \neq 0,|a| \leq|b|, c \geq 0$ \\
\hline$g_{6,36}$ & $2 \mathrm{ae}_{1}$ & $\mathrm{ae}_{2}+\mathrm{e}_{3}$ & $\mathrm{ae}_{3}$ & $\mathrm{be}_{4}+\mathrm{e}_{5}$ & $\mathrm{be}_{5}-\mathrm{e}_{4}$ & $b \geq 0$ \\
\hline$g_{6,37}$ & $2 \mathrm{ae}_{1}$ & $\mathrm{ae}_{2}+\mathrm{e}_{3}$ & $\mathrm{ae}_{3}-\mathrm{e}_{2}$ & $\mathrm{be}_{4}+\mathrm{ce}_{5}$ & $\mathrm{be}_{5}-\mathrm{ce}_{4}$ & $a \geq 0, c>0$ \\
\hline$g_{6,38}{ }^{*}$ & $2 \mathrm{ae}_{1}$ & $\mathrm{ae}_{2}+\mathrm{e}_{3}$ & $a e_{3}-e_{2}+e_{4}$ & $\mathrm{ae}_{4}+\mathrm{e}_{5}$ & $\mathrm{ae}_{5}-\mathrm{e}_{4}$ & $a \geq 0$ \\
\hline
\end{tabular}

Table 2: Lie algebras having nilradical isomorphic to $H \oplus \mathbb{R}^{2}:\left[\mathrm{e}_{2}, \mathrm{e}_{3}\right]=\mathrm{e}_{1}$.

\begin{tabular}{|c|c|c|c|c|c|c|}
\hline Algebra & {$\left[e_{1}, e_{6}\right]$} & {$\left[e_{2}, e_{6}\right]$} & {$\left[e_{3}, e_{6}\right]$} & {$\left[e_{4}, e_{6}\right]$} & {$\left[e_{5}, e 6\right]$} & Remarks \\
\hline $\operatorname{tg}_{6,39}$ & $(b+1) e_{1}$ & $(b+2) e_{2}$ & $a e_{3}$ & $b e_{4}$ & $e_{5}$ & $a \neq 0$ \\
\hline$g_{6,40}$ & $(a+1) e_{1}$ & $(a+2) e_{2}$ & $e_{2}+(a+2) e_{3}$ & $a e_{4}$ & $e_{5}$ & \\
\hline$g_{6,41}$ & $(a+1) e_{1}$ & $(a+2) e_{2}$ & $a e_{3}$ & $e 3+a e_{4}$ & $e_{5}$ & \\
\hline$g_{6,42}$ & $(a+1) e_{1}$ & $(a+2) e_{2}$ & $e_{3}$ & $a e_{4}$ & $e_{3}+e_{5}$ & \\
\hline$g_{6,43}$ & 0 & $e_{2}$ & $e_{2}+e_{3}$ & $-e_{4}$ & $e 3+e 5$ & \\
\hline $\operatorname{tg}_{6,44}$ & $2 e 1 a$ & $3 e 2$ & ae3 & e4 & $e 4+e 5$ & $a \neq 0$ \\
\hline$g_{6,45}$ & $2 e_{1}$ & $3 e_{2}$ & $e_{2}+3 e_{3}$ & $e_{4}$ & $e_{4}+e_{5}$ & \\
\hline$g_{6,46}$ & $2 e_{1}$ & $3 e_{2}$ & $e_{3}$ & $e_{3}+e_{4}$ & $e 4+e_{5}$ & \\
\hline $\operatorname{tg}_{6,47}$ & $e_{1}$ & $e_{2}$ & $a e_{3}$ & $\epsilon e_{2}+e_{4}$ & 0 & $a \neq 0, \epsilon=0, \pm 1$ \\
\hline$g_{6,48}$ & $e_{1}$ & $e_{2}$ & $e_{2}+e_{3}$ & $e_{4}$ & 0 & \\
\hline$g_{6,49}$ & $e_{1}$ & $e_{2}$ & 0 & $\epsilon e_{2}+e_{4}$ & $e_{3}$ & $\epsilon=0, \pm 1$ \\
\hline$g_{6,50}$ & $e_{1}$ & $e_{2}$ & $\epsilon_{e 2}+e_{3}$ & $e 3+e_{4}$ & 0 & $\epsilon=0, \pm 1$ \\
\hline $\operatorname{tg}_{6,51}$ & 0 & 0 & $e_{3}$ & $\epsilon e_{2}$ & 0 & $\epsilon= \pm 1$ \\
\hline$t g_{6,52}$ & 0 & 0 & $e_{3}$ & $\epsilon e_{2}$ & $e_{4}$ & $\epsilon=0, \pm 1$ \\
\hline
\end{tabular}

Table 3: Lie algebras having nilradical isomorphic to $\mathbb{R} \oplus A_{4,1}:\left[e_{1}, e_{5}\right]=e_{2},\left[e_{4}, e_{5}\right]=e_{1}$.

- Algebras that have two asterisks denote algebras in which there is a serious computational or merely typographical mistake

- Algebras that have three asterisks denote algebras which do not appear at all in Mubarakzyanov's list

- Algebras that have four asterisks denote algebras which are redundant 
Citation: Thompson G, Hettiarachchi C, Jones N, Shabanskaya A (2014) Representations of Six-dimensional Mubarakazyanov Lie algebras. J Generalized Lie Theory Appl 8: 211. doi: 10.4172/1736-4337.1000211

Page 4 of 10

\begin{tabular}{|c|c|c|c|c|c|c|}
\hline Algebra & {$\left[e_{1}, e_{6}\right]$} & {$\left[e_{2}, e_{6}\right]$} & {$\left[e_{3}, e_{6}\right]$} & {$\left[e_{4}, e_{6}\right]$} & {$\left[e_{5}, e_{6}\right]$} & Remarks \\
\hline$g_{6,53}$ & 0 & 0 & $e_{3}$ & $e_{4}$ & $-e_{5}$ & \\
\hline$g_{6,54}$ & $e_{1}$ & $a e_{2}$ & $(1-b) e_{3}$ & $(a-b) e_{4}$ & $b e_{5}$ & $|a| \leq 1$ \\
\hline$g_{6,55}$ & $e_{1}$ & $(1+a) e_{2}$ & $(1-a) e_{3}$ & $e_{1}+e_{4}$ & $a e_{5}$ & \\
\hline$g_{6,56}{ }^{*}$ & $e_{1}$ & 0 & $e_{2}$ & $-e_{4}$ & $e_{5}$ & \\
\hline$g_{6,57}$ & $e_{1}$ & $2 a e_{2}$ & $(1-a) e_{3}$ & $a e_{4}$ & $e_{4}+a e_{5}$ & \\
\hline$g_{6,58}$ & $3 e_{1}$ & $2 e_{2}$ & $e_{2}+2 e_{3}$ & $e_{4}$ & $e_{4}+e_{5}$ & \\
\hline$g_{6,59}{ }^{*}$ & $e_{1}$ & 0 & e3 & e2 & $\delta e_{4}$ & $\delta=0,1$ \\
\hline$g_{6,60}{ }^{*}$ & $e_{1}$ & $2 e_{2}$ & 0 & $e_{1}+e_{4}$ & $e_{4}+e_{5}$ & \\
\hline$g_{6,61}{ }^{*}$ & $2 e_{1}$ & 0 & $e_{3}$ & $-e_{4}$ & $e_{3}+e_{5}$ & \\
\hline$g_{6,62}{ }^{*}$ & $2 e_{1}$ & $e_{2}$ & $e_{2}+e_{3}$ & 0 & $e_{3}+e_{5}$ & \\
\hline$g_{6,63}$ & $e_{1}$ & $a e_{2}$ & $e_{3}$ & $e_{2}+a e_{4}$ & 0 & \\
\hline$g_{6,64}{ }^{*}$ & $e_{1}$ & $e_{2}$ & $\epsilon e_{2}+e_{3}$ & $e_{1}+e_{4}$ & 0 & $\epsilon= \pm 1$ \\
\hline$g_{6,65}{ }^{* *}$ & $a e_{1}+e_{2}$ & $a e_{2}$ & $(a-b) e_{3}+e_{4}$ & $(a-b) e_{4} b e_{5} a=1$ or $b=1$ & & \\
\hline$g_{6,66}$ & $2 e_{1}+e_{2}$ & $2 e_{2}$ & $e_{3}+e_{4}$ & $e_{4}$ & $e_{3}+e_{5}$ & \\
\hline$g_{6,67}^{* * * *}$ & $2 e_{1}+e_{2}$ & $2 e_{2}$ & $e_{3}+e_{4}$ & $e_{4}$ & $a e_{4}+e_{5}$ & equivalent to $g 6,65(a=2, b=1)$ \\
\hline$g_{6,68}^{*}$ & $e_{1}+e_{2}$ & $e_{2}$ & $e_{3}+e_{4}$ & $e_{1}+e_{4}$ & 0 & \\
\hline$g_{6,69}{ }^{* 00 * * *}$ & $e_{1}+e_{2}$ & $e_{2}$ & $e_{3}+e_{4}^{4}$ & $e_{2}+e_{4}$ & 0 & equivalent to $g_{6,65}(b=0)$ \\
\hline$g_{6,70^{* * *}}$ & $a e_{1}+e_{2}$ & $-e_{1}+a e_{2}$ & $\delta e_{2}+(a-b) e_{3}+e_{4}$ & $-e_{3}+(a-b) e_{4}$ & $b e_{5}$ & $\delta=0\left(g_{6,70}\right.$ in [1]) or $\delta=1$ and $b=0$ (new algebra) \\
\hline
\end{tabular}

Table 4: Lie algebras having nilradical isomorphic to $A_{5}, 1\left[e_{3}, e_{5}\right]=e 1\left[e_{4}, e_{5}\right]=e_{2}$.

In $g_{6,54}-g_{6,65}$, and $b$ are used in place of $\lambda$ and $y[1]$.

\begin{tabular}{|c|c|c|c|c|c|c|}
\hline Algebra & {$\left[e_{1}, e_{6}\right]$} & {$\left[e_{2}, e_{6}\right]$} & {$\left[e_{3}, e_{6}\right]$} & {$\left[e_{4}, e_{6}\right]$} & {$\left[e_{5}, e_{6}\right]$} & Remarks \\
\hline$g_{6,71}$ & $(a+3) e_{1}$ & $(a+2) e_{2}$ & $(a+1) e_{3}$ & $a e_{4}$ & $e_{5}$ & \\
\hline$g_{6,72}$ & $4 e_{1}$ & $3 e_{2}$ & $2 e_{3}$ & $e_{4}$ & $e_{4}+e_{5}$ & \\
\hline$g_{6,73-}{ }^{* * *}$ & $e_{1}$ & $e_{2}$ & $\epsilon e_{1}+e_{3}$ & $a e_{1}+\epsilon e_{2}+e_{4}$ & 0 & $\epsilon= \pm 1$, (if $a=0$, then $\left.g_{6,73}[1]\right)$ \\
\hline$g_{6,74}$ & $e_{1}$ & $e_{2}$ & $e_{3}$ & $e_{4}$ & 0 & \\
\hline$g^{6,75}$ & $e_{1}$ & $e_{2}$ & $e_{3}$ & $e_{1}+e_{4}$ & 0 & \\
\hline
\end{tabular}

Table 5: Lie algebras having nilradical isomorphic to $A_{5}, 2:\left[e_{2}, e_{5}\right]=e_{1},\left[e_{3}, e_{5}\right]=e_{2},\left[e_{4}, e_{5}\right]=e_{3}$.

\begin{tabular}{|c|c|c|c|c|c|c|}
\hline Algebra & {$\left[e_{1}, e_{6}\right]$} & {$\left[e_{2}, e_{6}\right]$} & {$\left[e_{3}, e_{6}\right]$} & {$\left[e_{4}, e_{6}\right]$} & {$\left[e_{5}, e_{6}\right]$} & Remarks \\
\hline$g_{6,76}$ & $(2 a+1) e_{1}$ & $(a+1) e_{2}$ & $(a+2) e_{3}$ & $e_{4}$ & $a e_{5}$ & $|a| \leq 1$ \\
\hline$g_{6,77}$ & $e_{1}$ & $e_{2}$ & $2 e_{3}$ & $\epsilon e_{1}+e_{4}$ & 0 & \\
\hline$g_{6,78}$ & $-e_{1}$ & 0 & $e_{3}$ & $e_{3}+e_{4}$ & $-e_{5}$ & \\
\hline$g_{6,79}$ & $3 e_{1}$ & $2 e_{2}$ & $e_{1}+3 e_{3}$ & $e_{4}+e_{5}$ & $e_{5}$ & \\
\hline$g_{6,80}^{\prime}{ }^{* * *}$ & $3 a e_{1}+e_{3}$ & $2 a e_{2}$ & $3 a e_{3}-e_{1}$ & $a e_{4}-e_{5}$ & $e_{4}+a e_{5}$ & \\
\hline$g_{6,81}^{\prime}{ }^{* * *}$ & $e_{3}$ & 0 & $-e_{1}$ & $\epsilon e_{1}-e_{5}$ & $e_{4}$ & $\epsilon= \pm 1$ \\
\hline$g_{6,80}{ }^{* * * *}$ & $2 e_{1}$ & $e_{2}$ & $e_{3}$ & 0 & $e_{5}$ & equivalent to $g_{6,76}(a=0)$ \\
\hline$g_{6,81}{ }^{*}$ & $2 e_{1}$ & $e_{2}$ & $e_{3}$ & 0 & $\epsilon e_{3}+e_{5}$ & $\epsilon= \pm 1$ equivalent to $g_{6,77}$ \\
\hline
\end{tabular}

Table 6: Lie algebras having nilradical isomorphic to $A_{5}, 3:\left[e_{2}, e_{4}\right]=e_{3},\left[e_{2}, e_{5}\right]=e_{1},\left[e_{4}, e_{5}\right]=e_{2}$.

\begin{tabular}{|c|c|c|c|c|c|c|}
\hline Algebra & {$\left[e_{1}, e_{6}\right]$} & {$\left[e_{2}, e_{6}\right]$} & {$\left[e_{3}, e_{6}\right]$} & {$\left[e_{4}, e_{6}\right]$} & {$\left[e_{5}, e_{6}\right]$} & Remarks \\
\hline$g_{6,82} \lambda=a, \lambda_{1}=b$ & $2 \delta e_{1}$ & $(\delta+a) e_{2}$ & $(\delta+b) e_{3}$ & $(\delta-a) e_{4}$ & $(\delta-b) e_{5}$ & $\delta=1,0 \leq a \leq b$ or $\delta=0,0 \leq a \leq 1, b=1$ \\
\hline$g_{6,83} \lambda=a$ & $2 \delta e_{1}$ & $(\delta+a) e_{2}+e_{3}$ & $(\delta+a) e_{3}$ & $(\delta-a) e 4$ & $(\delta-a) e 5-e 4$ & $\delta=1,0 \leq a$ or $\delta=0, a=1$ \\
\hline$g_{6,84}$ & 0 & $e_{2}$ & 0 & $-e_{4}$ & $e_{3}$ & \\
\hline$g_{6,85} \lambda=a$ & $2 e_{1}$ & $(a+1) e_{2}$ & $e_{3}$ & $(1-a) e_{4}$ & $e_{3}+e_{5}$ & $a \geq 0$ \\
\hline$g_{6,86}{ }^{* * * *}$ & $2 e_{1}$ & $e_{2}+e 3$ & $e_{3}$ & $e_{4}$ & $e_{5}$ & equivalent to $g_{6,83}$ for $\alpha=2, \lambda=0$ \\
\hline$g_{6,87}^{* *}$ & $2 e_{1}$ & $e_{2}+e_{5}$ & $e_{3}+e_{4}$ & $e_{4}$ & $e_{3}+e_{5}$ & \\
\hline$g_{6,88}{ }^{*} \mu_{0}=a, v_{0}=1$ & $\alpha e_{1}$ & $\left(\frac{\alpha}{2}+a\right) e_{2}+e_{3}$ & $\left(\frac{\alpha}{2}+a\right) e_{3}-e_{2}$ & $\left(\frac{\alpha}{2}-a\right) e_{4}+e_{5}$ & $\left(\frac{\alpha}{2}-a\right) e_{5}-e_{4}$ & $a \geq 0$ \\
\hline$g_{6,89}{ }^{*} s=a, v_{0}=b$ & $2 \delta e_{1}$ & $(\delta+a) e_{2}$ & $\delta e_{3}+b e_{5}$ & $(\delta-a) e_{4}$ & $\delta e_{5}-b e_{3}$ & $\begin{aligned} \delta=1, a \geq 0 \text { and } b>0 \text { or } \delta & =0 \text { and } a=1, b>0 \text { or } a=0, b \\
& =1\end{aligned}$ \\
\hline$g_{6,90}{ }^{* *} v_{0}=a$ & $2 \delta e_{1}$ & $\delta e_{2}$ & $\delta e_{3}+a e_{5}$ & $e_{2}+\delta e_{4}$ & $\delta e_{5}-a e_{3}$ & $\delta=1, a /=0$ or $\delta=0, a= \pm 1$ \\
\hline$g_{6,91}{ }^{* *, * * * *}$ & 0 & 0 & $e_{5}$ & $e_{2}$ & $-e 3$ & included in $g_{6,90}$ as $\delta=0, a=1$ \\
\hline$g_{6,92}^{\prime}{ }^{*, * * *} s=a, v_{0}=b$ & $2 \delta e_{1}$ & $\delta e_{2}+a e_{4}$ & $\delta e_{3}+b e_{5}$ & $\delta e_{4}-a e_{2}$ & $\delta e_{5}-b e_{3}$ & $\begin{array}{c}\delta=1,0<a \leq|b| \text { or } \delta=0, a=1,0<|b| \leq 1 \text { are new } \\
\text { algebras }\end{array}$ \\
\hline$g_{6,92}{ }^{* * * *} v_{0}=a, \mu_{0}=b$ & $2 \delta e_{1}$ & $\delta e_{2}+a e_{3}$ & $\delta e_{3}-b e_{2}$ & $\delta e_{4}+b e_{5}$ & $\delta e_{5}-a e_{4}$ & equivalent to cases of $g_{6,82}, g_{6,83}$ or $g_{6,88}$ \\
\hline$g_{6,93}{ }^{* *} v_{0}=a$ & $2 \delta e_{1}$ & $\delta e_{2}+e_{4}+a e_{5}$ & $\delta e_{3}+a e_{4}$ & $\delta e_{4}-a e_{3}$ & $\delta e_{5}-a e_{2}-e_{3}$ & $\delta=1, a \geq 0$ or $\delta=0, a=1$ \\
\hline
\end{tabular}

Table 7 : Lie algebras having nilradical isomorphic to $A 5,4:\left[e_{2}, e_{4}\right]=e 1,\left[e_{3}, e_{5}\right]=e_{1}$. 
Citation: Thompson G, Hettiarachchi C, Jones N, Shabanskaya A (2014) Representations of Six-dimensional Mubarakazyanov Lie algebras. J Generalized Lie Theory Appl 8: 211. doi: 10.4172/1736-4337.1000211

Page 5 of 10

\begin{tabular}{|c|c|c|c|c|c|c|}
\hline Algebra & {$\left[e_{1}, e_{6}\right]$} & {$\left[e_{2}, e_{6}\right]$} & {$\left[e_{3}, e_{6}\right]$} & {$\left[e_{4}, e_{6}\right]$} & $2 e_{4}$ \\
\hline$g_{6,94}$ & $(a+2) e_{1}$ & $(a+1) e_{2}$ & $a e_{3}$ & $2 e_{4}$ & $e_{5}$ \\
\hline$g_{6,94}$ & $(a+2) e_{1}$ & $(a+1) e_{2}$ & $a e 3$ & $2 e_{4}$ & $e_{5}$ \\
\hline$g_{6,94}$ & $(a+2) e_{1}$ & $(a+1) e_{2}$ & $a e_{3}$ & $2 e_{4}$ & $e_{5}$ \\
\hline$g_{6,97}$ & $4 e_{1}$ & $3 e_{2}$ & $2 e_{3}+e_{4}$ & $e_{3}$ & $\delta e_{4}$ \\
\hline$g_{6,98}$ & ${ }^{*} e_{1}$ & $\delta e_{1}+e_{2}$ & $e_{3}$ & $\delta=0,1$ \\
\hline
\end{tabular}

Table 8: Lie algebras having nilradical isomorphic to $A 5,5:\left[e_{3}, e_{4}\right]=e_{1},\left[e_{2}, e_{5}\right]=e_{1},\left[e_{3}, e_{5}\right]=e_{2}$.

\begin{tabular}{|c|c|c|c|c|c|c|c|c|c|c|c|c|c|}
\hline Algebra & {$\left[e_{3}, e_{4}\right]$} & {$\left[e_{2}, e_{5}\right]$} & {$\left[e_{3}, e_{5}\right]$} & {$\left[e_{4}, e_{5}\right]$} & {$\left[e_{1}, e_{6}\right]$} & {$\left[e_{2}, e_{6}\right]$} & {$\left[e_{3}, e_{6}\right]$} & {$\left[e_{4}, e_{6}\right]$} & {$\left[e_{5}, e_{6}\right]$} & Remarks \\
\hline$g_{6,99}$ & $e_{1}$ & $e_{1}$ & $e_{2}$ & $e_{3}$ & $5 e_{1}$ & $4 e_{2}$ & $3 e_{3}$ & $2 e_{4}$ & $e_{5}$ & \\
\hline
\end{tabular}

Table 9: Lie algebras having nilradical isomorphic to $A 5,6$.

$g_{6,1}-s_{6}, 0,0,0,0, s_{1}, 0,-a s_{6}, 0,0,0, s_{2}, 0,0,-b s_{6}, 0,0, s_{3}, 0,0,0,-c s_{6}, 0, s_{4}, 0,0,0,0,-d s_{6}, s_{5}, 0,0,0,0,0,0$

$g_{6,2} a s_{6},-s_{6}, 0,0,0, s_{1}, 0,-a s_{6}, 0,0,0, s_{2}, 0,0,-s_{6}, 0,0, s_{3}, 0,0,0,-c s_{6}, 0, s_{4}, 0,0,0,0,-d s_{6}, s_{5}, 0,0,0,0,0,0$

$g_{6,3} \quad-a_{s 6},-s_{6}, 0,0,0, s_{1}, 0,-a s_{6},-s_{6}, 0,0, s_{2}, 0,0,-a s_{6}, 0,0, s_{3}, 0,0,0,-s_{6}, 0, s_{4}, 0,0,0,0,-d s_{6}, s_{5}, 0,0,0,0,0,0$

$g_{6,4}-a s_{6},-s_{6}, 0,0,0, s_{1}, 0,-a s_{6},-s_{6}, 0,0, s_{2}, 0,0,-a s_{6},-s_{6}, 0, s_{3}, 0,0,0,-a s_{6}, 0, s_{4}, 0,0,0,0,-b s_{6}, s_{5}, 0,0,0,0,0,0$

$g_{6,5}-s_{6},-s_{6}, 0,0,0, s_{1}, 0,-s_{6},-s_{6}, 0,0, s_{2}, 0,0,-s_{6},-s_{6}, 0, s_{3}, 0,0,0,-s_{6},-s_{6}, s_{4}, 0,0,0,0,-s_{6}, s_{5}, 0,0,0,0,0,0$

$g_{6,6} \quad-a s_{6},-s_{6}, 0,0,0, s_{4}, 0,-a s_{6}, 0,0,0, s_{5}, 0,0,-b s_{6},-s_{6}, 0, s_{2}, 0,0,0,-b s_{6}, 0, s_{3}, 0,0,0,0,-s_{6}, s_{1}, 0,0,0,0,0,0$

$g_{6,7}-b s_{6},-s_{6}, 0,0,0, s_{5}, 0,-b s_{6}, 0,0,0,-s_{4}, 0,0,-a s_{6},-s_{6}, 0, s_{1}, 0,0,0,-a s_{6},-s_{6}, s_{2}, 0,0,0,0,-a s_{6}, s_{3}, 0,0,0,0,0,0$

$g_{6,8}-d s_{6},-s_{6}, 0,0,0, s_{5}, s_{6},-d s_{6}, 0,0,0, s_{4}, 0,0,-a s_{6}, 0,0, s_{2}, 0,0,0,-b s_{6}, 0, s_{3}, 0,0,0,0,-c s_{6}, s_{1}, 0,0,0,0,0,0$

$g_{6,9} \quad-c s_{6},-s_{6}, 0,0,0, s_{4}, s_{6},-c s_{6}, 0,0,0, s_{5}, 0,0,-b s_{6},-s_{6}, 0, s_{2}, 0,0,0,-b s_{6}, 0, s_{3}, 0,0,0,0,-a s_{6}, s_{1}, 0,0,0,0,0,0$

$g_{6,10}-a s_{6},-s_{6}, 0,0,0, s_{1}, 0,-a s_{6},-s_{6}, 0,0, s_{2}, 0,0,-a s_{6}, 0,0, s_{3}, 0,0,0,-b s_{6}, s_{6},-s_{4}, 0,0,0,-s_{6},-b s_{6}, s_{5}, 0,0,0,0,0,0$

$g_{6,11}-a s_{6}, 0,0,0,0, s_{1}, 0,-b s_{6},-s_{6}, 0,0, s_{2}, 0, s_{6},-b s_{6}, 0,0, s_{3}, 0,0,0,-c s_{6},-d s_{6}, s_{5}, 0,0,0, d s_{6},-c s_{6},-s_{4}, 0,0,0,0,0,0$

$g_{6,12}-a s_{6}, 0,0,0,0, s_{1}, 0,-b s_{6},-s_{6},-s_{6}, 0, s_{3}, 0, s_{6},-b s_{6}, 0,-s_{6},-s_{2}, 0,0,0,-b s_{6},-s_{6}, s_{5}, 0,0,0, s_{6},-b s_{6},-s_{4}, 0,0,0,0,0,0$

$g_{6,13}-(a+b) s_{6},-s_{3}, s_{2}, 0,0,2 s_{1}, 0,-a s_{6}, 0,0,0, s_{2}, 0,0,-b s_{6}, 0,0, s_{3}, 0,0,0,-s_{6}, 0, s_{4}, 0,0,0,0,-c s_{6}, s_{5}, 0,0,0,0,0,0$

$g_{6,14}$ $-(a+b) s_{6},-s_{2}, s_{3},-2 s_{6}, 0,-2 s_{1}, 0,-a s_{6}, 0,0,0, s_{3}, 0,0,-b s_{6}, 0,0, s_{2}, 0,0,0,-(a+b) s_{6}, 0, s_{5}, 0,0,0,0,-s_{6}, s_{4}, 0,0,0,0,0,0$

$g_{6,15}-(a+1) s_{6},-s_{2}, s_{3}, 0,0,-s_{1}, 0,-s_{6}, 0,0,0, s_{3}, 0,0,-a s_{6}, 0,0, a s_{2}, 0,-s_{6}, 0,-s_{6}, 0, s_{3}+s_{5}, 0,0,-s_{6}, 0,-a s_{6}, s_{2}+a s_{4}, 0,0,0,0,0,0$ $0,0,0,0,-s_{6},-1$

$2 s_{5}, 0,-s_{6},-s_{6},-1$

$g_{6,16} \quad 2 s_{3},-s_{2}, s_{1}, 0,0,-s_{6},-s_{6}, 0, s_{4}, 0,0,0,-s_{6}, 0, s_{2}, 0,0,0,0,0,-1$ $2 s_{3}, 0,0,0,0,0,0$

$g_{6,17.1}-s_{6}, 0,0,0,0, s_{5}, 0,-a s_{6}, 0,-s_{6},-s_{3}, s_{1}, 0,0,-a s_{6}, 0,0,-s_{3}, 0,0,0,0,-s_{6}, s_{4}, 0,0,0,0,0, s_{2}, 0,0,0,0,0,0$

$g_{6,17.2} \quad 0, s_{3}, 0,0,0,-s_{4}, 0,0,0,0,0,-s_{6}, 0,0,-s_{6}, s_{3},-s_{6},-s_{1}, 0,0,0,-s_{6}, 0, s_{2}, 0,0,0,0,-s_{6},-s_{5}, 0,0,0,0,0,0$

$g_{6,18}-b s_{6}, 0,0,0,0, s_{5}, 0,-s_{6}, 0,-s_{6}, 0, s_{4}, 0,0,-(a+1) s_{6}, 0, s_{3},-s_{1}, 0,0,0,-s_{6}, 0, s_{3}, 0,0,0,0,-a s_{6}, s_{2}, 0,0,0,0,0,0$

$g_{6,19}-(a+1) s_{6}, 0,-s_{3}, s_{2},-s_{6}, 2 s_{1}, 0,-s_{6}, 0,-s_{6}, 0, s_{4}, 0,0,-a s_{6}, 0,0, s_{2}, 0,0,0,-s_{6}, 0, s_{3}, 0,0,0,0,-(a+1) s_{6}, 2 s_{5}, 0,0,0,0,0,0$

$g_{6,20}-b s_{6}, 0,0,0,0, s_{5}, 0,-s_{6},-s_{6}, s_{2},-s_{3}, s_{1}, 0,0,-s_{6},-s_{6}, 0, s_{4}, 0,0,0,-s_{6}, 0, s_{3}, 0,0,0,0,0,0,0,0,0,0,0,0$

$g_{6,21}-2 a s_{6}, a s_{2}, s_{2}-s_{3}, 0,0,2 s_{1}, 0,-a s_{6},-s_{6}, 0,0, s_{3}, 0,0,-a s_{6}, 0,0, a s_{2}, 0,0,0,-s_{6}, 0, s_{5}, 0,0,0,0,-b s_{6}, s_{4}, 0,0,0,0,0,0$

$g_{6,22}-s_{6}, 0,0,0,0, s_{4}, 0,-2 a s_{6}, s_{2},-s 3,2 \in s_{6}, 2 s_{1}, 0,0,-a s_{6},-a s_{6}, 0, s_{3}, 0,0,0,-a s_{6}, 0, s_{2}, 0,0,0,0,-2 a s_{6},-s_{5}, 0,0,0,0,0,0$

$g_{6,23.1}-2 s_{6}, 0, s_{2},-s_{3}, 2 s_{6}, 2 s_{1}, 0,-s_{6},-s_{6}, 0,0, s_{3}+s_{4}, 0,0,-s_{6},-s_{6}, 0, s_{2}+s_{3}, 0,0,0,-s_{6}, 0, s_{2}, 0,0,0,0,-2 s_{6},-s_{5}, 0,0,0,0,0,0$

$g_{6,23.2}-(2 \delta+a) s_{6}, 0,0,0,0, s_{5}, 0,-2 \delta s_{6}, 0, s_{2}, s_{2}-s_{3}, s_{1}, 0,0,-\delta s_{6},-s_{6}, 0, s_{4}, 0,0,0,-\delta s_{6},-s_{6}, s_{3}, 0,0,0,0,-\delta s_{6}, s_{2}, 0,0,0,0,0,0$

$g_{6,24}-s_{6}, 0,0,0,0, s_{5}, 0,0,-2 s_{6}, s_{2},-s_{3},-2 s_{1}, 0,0,0,-s_{6}, 0,-s_{4}, 0,0,0,0,-s_{6},-s_{3}, 0,0,0,0,0,-s_{2}, 0,0,0,0,0,0$

$g_{6,25}-b s_{6}, s_{4}, 0,0,0,-s_{5}, 0,0,0,0,0,-s_{6}, 0,0,-(a+1) s_{6}, 0, s_{3},-s_{1}, 0,0,0,-a s_{6}, 0, s_{3}, 0,0,0,0,-s_{6}, s_{2}, 0,0,0,0,0,0$

$g_{6,26}-a s_{6},-s_{6}, 0,0, s_{2}, s_{1}, 0,-a s_{6},-s_{6}, 0,0, s_{5}, 0,0,-a s_{6}, 0,0, s_{4}, 0,0,0,-s_{6}, 0, s_{2}, 0,0,0,0,(1-a) s_{6}, s_{3}, 0,0,0,0,0,0$

$g_{6,27}-(a+b) s_{6}, \delta s_{6}, 0,-s_{2},-s_{1}, 0,-b s_{6},-s_{6}, 0, s_{5}, 0,0,-b s_{6},-s_{6}, s_{4}, 0,0,0,-b s_{6}, s_{3}, 0,0,0,0,0$

$g_{6,28}-2 s_{6}, s_{2},-s_{3}, 0,0,2 s_{1}, 0,-s_{6},-s_{6}, 0,0, s_{3}, 0,0,-s_{6}, 0,0, s_{2}, 0,0,0,-b s_{6},-s_{6}, s_{5}, 0,0,0,0,-b s_{6}, s_{4}, 0,0,0,0,0,0$

$g_{6,29} \quad 2 s_{3}, s_{6},-s_{6}, 0, s_{1}, 0,-s_{6},-s_{6}, 0,0,1$

$2 s_{3}, 0,0,-s_{6}, 0,0, s_{2}, 0,0,0,-2 s_{6},-s_{6}, s_{5}, 0,0,0,0,-2 s_{6}, s_{4}, 0,0,0,0,0,0$

$g_{6,30}-s_{6}, 0,0, s_{4},-s_{5}, 0,0, s_{2}, 0,-s_{1}, 0,0,0, s_{2},-s_{3}, 0,0,0,0,-s_{6}, 0,0,0,0,0$

$-2 s_{6}, 0,0,3$

$2 s_{2},-3$

$2 s_{3}-3 s_{6}, 9$

$2 s_{1}, 0,-s_{6},-s_{6}, 0,0,3$

$g_{6,31} \quad 2 s_{5}, 0,0,-s_{6},-s_{6}, 0,3$

$2 s_{4}, 0,0,0,-s_{6},-s_{6}, 3$

$2 s_{3}, 0,0,0,0,-s_{6}, 3$

$2 s_{2}, 0,0,0,0,0,0$

$g_{6,32}-c s_{6}, 0,0,0,0, s_{5}, 0,-b s_{6}, 0,0,0, s_{4}, 0,0,-2 a s_{6},-s_{2}, s_{3},-2 s_{1}, 0,0,0,-a s_{6},-s_{6}, s_{3}, 0,0,0, s_{6},-a s_{6}, s_{2}, 0,0,0,0,0,0$

$g_{6,33}-b s_{6}, 0,0,0,0, s_{5}, 0,-2 a s_{6},-s_{3}, s_{2},-s_{6}, 2 s_{1}, 0,0,-a s_{6}, s_{6}, 0, s_{2}, 0,0,-s_{6},-a s_{6}, 0, s_{3}, 0,0,0,0,-2 a s_{6}, 2 s_{4}, 0,0,0,0,0,0$ 
Citation: Thompson G, Hettiarachchi C, Jones N, Shabanskaya A (2014) Representations of Six-dimensional Mubarakazyanov Lie algebras. J Generalized Lie Theory Appl 8: 211. doi: 10.4172/1736-4337.1000211

Page 6 of 10

$g_{6,34}-2 a s_{6},-s_{3}, s_{2},-\delta s_{6}, 0,2 s_{1}, 0,-a s_{6}, s_{6}, 0,0, s_{2}, 0,-s_{6},-a s_{6}, 0,0, s_{3}, 0,0,0,-(2 a+b) s_{6},-s_{6}, 2 s_{5}, 0,0,0,0,-(2 a+b) s_{6}, 2 s_{4}, 0,0,0,0,0,0$

$g_{6,35}-c s_{6}, s_{6}, 0,0,0, s_{4},-s_{6},-c s_{6}, 0,0,0, s_{5}, 0,0,-(a+b) s_{6},-s_{3}, s_{2}, 2 s_{1}, 0,0,0,-a s_{6}, 0, s_{2}, 0,0,0,0,-b s_{6}, s_{3}, 0,0,0,0,0,0$

$g_{6,36}-c s_{6}, s_{6}, 0,0,0, s_{4},-s_{6},-c s_{6}, 0,0,0, s_{5}, 0,0,-2 a_{s 6},-s_{2}, s_{3},-2 s_{1}, 0,0,0,-a s_{6},-s_{6}, s_{3}, 0,0,0,0,-a s_{6}, s 2,0,0,0,0,0,0$

$g_{6,37}-a 2 s_{6},-s_{2}, s_{3}, 0,0,-2 s_{1}, 0,-a s_{6},-s_{6}, 0,0, s_{3}, 0, s_{6},-a s_{6}, 0,0, s_{2}, 0,0,0,-b s_{6},-c s_{6}, s_{5}, 0,0,0, c s_{6},-b s_{6}, s_{4}, 0,0,0,0,0,0$

$g_{6,38}-2 a s_{6}, 0,0,-s_{3},-s_{2},-2 s_{1}, 0,-a s_{6},-s_{6},-s_{6}, 0,-s_{3}+2 s_{5}, 0, s_{6},-a s_{6}, 0,-s_{6}, 2 s_{4}, 0,0,0,-a s_{6},-s_{6},-s_{2}, 0,0,0, s_{6},-a s_{6}, s_{3}, 0,0,0,0,0,0$

$g_{6,39}(b-a+2) s_{6}, 0,0,0, s 3,0,0, s 5,0, s 2,0,0, s 6, s 5,-s 1,0,0,0,2 s 6, s 4,0,0,0,0,(b+2) s 6$

$g_{6,40} \quad 0, s 5,0, s 3, s 2,0, s 6, s 5,0,-s 1,0,0,2 s 6,0, s 4,0,0,0,(a+2) s 6, s 6,0,0,0,0,(a+2) s 6$

$g_{6,41}-(2+a) s 6, s 5,0,0, s 1, s 2,0,-(a+1) s 6,0, s 5,-s 4,-s 1,0,0,-a s 6, s 6,0,-s 3,0,0,0,-a s 6,0, s 4,0,0,0,0,-s 6,0,0,0,0,0,0,0$ $-s 6,0,0, s 6,0,-s 3,0,-(a+2) s 6,1$

$g_{6,42} \quad 4 s 5,-1$

$4 s 1,0,-s 2,0,0,-(a+1) s 6, s 4,-2 s 5,3 s 1,0,0,0,-s 6,0, s 5,0,0,0,0,-a s 6, s 4,0,0,0,0,0,0$

$-s 6,-s 5,-s 6,0, s 1, s 2,0,0,0,-s 5, s 4,0,0,0,-s 6,0,-s 6, s 3,0,0,0, s 6,0,-s 4,0,0,0,0,-s 6, s 5,0,0,0,0,0,0-s 6,-s 5,-s 6,0, s 1, s 2,0,0,0,-s 5, s 4,0,0$, $0,-s 6,0,-s 6, s 3,0,0,0, s 6,0,-s 4,0,0,0,0,-s 6, s 5,0,0,0,0,0,0$

$g_{644} \quad 3 s 6,-s 5,0,0, s 1,3 s 2,0,2 s 6,0,-s 5, s 4-s 5,2 s 1,0,0, a s 6,0,0, s 3,0,0,0, s 6, s 6, s 4,0,0,0,0, s 6, s 5,0,0,0,0,0,0$

$g_{6,45} \quad 3 s 6,-s 5, s 6,0, s 1,3 s 2,0,2 s 6,0,-s 5, s 4-s 5,2 s 1,0,0,3 s 6,0,0,3 s 3,0,0,0, s 6, s 6, s 4,0,0,0,0, s 6, s 5,0,0,0,0,0,0$

$g_{6,46} 3 s 6,-s 5,0,0, s 1,3 s 2,0,2 s 6,0,-s 5, s 4-s 5,2 s 1,0,0, s 6, s 6,0, s 3,0,0,0, s 6, s 6, s 4,0,0,0,0, s 6, s 5,0,0,0,0,0,0$

$g_{6,47}-s 6, s 5,0,-\epsilon s 6,-s 1, s 2,0,-s 6,0, s 5, s 4,-s 1,0,0,-a s 6,0,0, s 3,0,0,0,-s 6,0, s 4,0,0,0,0,0,0,0,0,0,0,0,0$

$g_{6,48}-s 6, s 5,-s 6,0,-s 1, s 2,0,-s 6,0, s 5, s 4,-s 1,0,0,-s 6,0,0, s 3,0,0,0,-s 6,0, s 4,0,0,0,0,0,0,0,0,0,0,0,0$

$g_{6,49} \quad 0,0,0,-s 6,0, s 3,0,-s 6,-s 5, s 1,-s 6,2 s 2,0,0,-s 6, s 4,0, s 1,0,0,0,0,0, s 5,0,0,0,0,-s 6,2 \in s 4,0,0,0,0,0,0$

$g_{6,50}-s 6, s 5,-\epsilon s 6,0,-s 1, s 2,0,-s 6,0, s 5, s 4,-s 1,0,0,-s 6,-s 6,0, s 3,0,0,0,-s 6,0, s 4,0,0,0,0,0,0,0,0,0,0,0,0$

$g_{6,51}-s 6,0,0,0,0, s 3,0,0, s 5,0, s 4, s 2,0,0,0, s 5,0,-s 1,0,0,0,0,0, s 4,0,0,0,0,0, \in s 6,0,0,0,0,0,0$

$g_{6,52}-\epsilon s 6,0,0,0,0, s 3,0,0,2 s 5,-s 6, s 4,2 s 2,0,0,0, s 5,0,-s 1,0,0,0,0, s 5, s 4,0,0,0,0,0, s 6,0,0,0,0,0,0$

$g_{6.53} s 6,0, s 5,0,-s 2,0, s 6,0, s 5,-s 1,0,0,0,0, s 4,0,0,0,0, s 3,0,0,0,0, s 6$

$g_{6,54}-a s 6,0, s 5,0,-s 2,0,-s 6,0, s 5,-s 1,0,0,(b-a) s 6,0, s 4,0,0,0,(b-1) s 6, s 3,0,0,0,0,0$

$-(a+1) s 6,0,0,0, s 5,-1$

$2 s 2,0,-s 6,1$

$g_{6,55} \quad 2 s 3, s 5, s 6,-1$

$2 s 1,0,0$-as6, 0, 0, s5, 0, 0, 0, (a-1)s6, 0, s3, 0, 0, 0, 0,-s6, 1

$2 s 4,0,0,0,0,0,0$

$g_{6,56}-s 6,0, s 5,0,-s 1,0,0,-s 6, s 5, s 2,0,0,0,0, s 3,0,0,0, s 6,-s 4,0,0,0,0,0$

$g_{6,57}-s 6, s 5,0,0,0,-s 1,0,(a-1) s 6,0,0,0, s 3,0,0,-2 a s 6, s 5,0, s 2,0,0,0,-a s 6, s 5,-s 4,0,0,0,0,0,-s 6,0,0,0,0,0,0$ $-3 s 6,0,-s 5,0,1$

$g_{6,58} \quad 2 s 3,3$

$2 s 1,0,-2 s 6,-s 6,-s 5, s 4-s 5,2 s 2,0,0,-2 s 6,0,0, s 3,0,0,0,-s 6,-s 6, s 4,0,0,0,0,-s 6, s 5,0,0,0,0,0,0$

$g_{6,59}-s 6, s 5,0,0,0,-s 1,0,-s 6,0,0,0, s 3,0,0,0,-s 5-s 6,0,-s 2,0,0,0,0,-s 5-s 6,-s 4,0,0,0,0,0,-\delta s 5,0,0,0,0,0,0$

$g_{6,60}-s 6,0,-s 5,-s 6, s 3, s 1,0,-2 s 6,0,-s 5, s 4+s 5,2 s 2,0,0,0,0,0,0,0,0,0,-s 6,-s 6, s 4,0,0,0,0,-s 6, s 5,0,0,0,0,0,0$

$g_{6,61} \quad 0,0,0,0, s 4, s 2,0, s 6,0,0,0, s 4,0,0,-2 s 6,-s 5, s 3,2 s 1,0,0,0,-s 6,-s 6, s 3,0,0,0,0,-s 6, s 5,0,0,0,0,0,0$ $2 s 6,0,-s 5, s 3-1$

$2 s 5,2 s 1,0, s 6, s 6,1$

$g_{6,62} \quad 4 s 4,3 s 2,0,0, s 6,-1$

$2 s 6, s 3,0,0,0, s 6, s 5,0,0,0,0,0$

$g_{6,63}-a s 6,-s 5-s 6,0,0,0, s 2,0,-a s 6,0,0,0, s 4,0,0,-s 6, s 5, s 3,-s 1,0,0,0,-s 6,0, s 3,0,0,0,0,0,0,0,0,0,0,0,0$

$g_{6,64}-s 6,0, s 5,-s 6,-s 3, s 1,0,-s 6,-\epsilon s 6, s 5, s 4,-s 2,0,0,-s 6,0,0,-s 3,0,0,0,-s 6,0, s 4,0,0,0,0,0,0,0,0,0,0,0,0$

$g_{6,65} \quad-a s 6,0, s 6,0,-s 4,-s 2,0,(b-a) s 6,0, s 6,0,-s 4,0,0,-a s 6,0, s 3, s 1,0,0,0,(b-a) s 6,0, s 3,0,0,0,0,-b s 6, s 5,0,0,0,0,0,0$

$g_{6,66}-2 s 6,-s 6,-s 5,0,-s 3+s 4+s 5,-s 1+2 s 2,0,-2 s 6,0,-s 5, s 3-s 5,2 s 1,0,0,-s 6,-s 6,0, s 4,0,0,0,-s 6,-s 6, s 3,0,0,0,0,-s 6, s 5,0,0,0,0,0,0$

$g_{6,68}-s 6,-s 6,-s 5,0,-s 3+s 4, s 2,0,-s 6,-s 6,-s 5, s 3, s 1,0,0,-s 6,-s 6,0, s 4,0,0,0,-s 6,0, s 3,0,0,0,0,0,0,0,0,0,0,0,0$

$-a s 6, s 6, s 5,0,-s 3$, as $1-s 2,-s 6,-a s 6, \delta s 6, s 5,-s 4, s 1+a s 2+\delta s 3,0,0,(b-a) s 6, s 6,0,(b-a) s 3+s 4,0,0,-s 6,(b-a) s 6,0,-s 3+(b-a) s 4,0,0,0,0,-b s 6,-$ bs5, 0, 0, 0, 0, 0, 0

$g_{6,71}-(a+3) s 6, s 5,0,0,-s 1,0,-(a+2) s 6, s 5,0, s 2,0,0,-(a+1) s 6, s 5,-s 3,0,0,0,-a s 6, s 4,0,0,0,0,0$

$g_{6,72}-4 s 6,-s 5,0,0, s 2,4 s 1,0,-3 s 6,-s 5,0, s 3,3 s 2,0,0,-2 s 6,-s 5, s 4-s 5,2 s 3,0,0,0,-s 6,-s 6, s 4,0,0,0,0,-s 6, s 5,0,0,0,0,0,0$

$g_{6,73}-s 6,-s 5,-\epsilon s 6,-a s 6, s 2-\epsilon s 4, s 1,0,-s 6,-s 5,-\epsilon s 6, s 3, s 2,0,0,-s 6,-s 5, s 4, s 3,0,0,0,-s 6,0, s 4,0,0,0,0,0,0,0,0,0,0,0,0$

$g_{6,74}-s 6, s 5,0,0,-s 2, s 1,0,-s 6, s 5,0, s 3,-s 2,0,0,-s 6, s 5,-s 4, s 3,0,0,0,-s 6,0,-s 4,0,0,0,0,0,0,0,0,0,0,0,0$

$g_{6,75}-s 6,-s 5,0,-s 6, s 2, s 1,0,-s 6,-s 5,0, s 3, s 2,0,0,-s 6,-s 5, s 4, s 3,0,0,0,-s 6,0, s 4,0,0,0,0,0,0,0,0,0,0,0,0$ $-(a+2) s 6,0,1$

$g_{6,76} \quad 2 s 4,1$

$2 s 2,-s 3,0,-(2 a+1) s 6, s 5,0,-s 1,0,0,-(a+1) s 6, s 5, s 2,0,0,0,-s 6,-s 4,0,0,0,0,0$

$g_{6,77}-s 6,0, s 5,-\epsilon s 6,-s 2, s 1,0,-2 s 6,-s 4,-s 2,0,-2 s 3,0,0,-s 6, s 5, s 4,-s 2,0,0,0,-s 6,0, s 4,0,0,0,0,0,0,0,0,0,0,0,0$

$g_{6,78} s 6,0,-s 5,0, s 2,-s 1,0,-s 6,-s 4, s 2-s 6,0, s 3,0,0,0,-s 5, s 4,0,0,0,0,-s 6,0, s 4,0,0,0,0, s 6,-s 5,0,0,0,0,0,0$

$g_{6,79}-3 s 6,-s 6, s 4-s 5, s 2,0,3 s 1-2 s 3,0,-3 s 6,-s 4,0, s 2,3 s 3,0,0,-2 s 6, s 4, s 4-s 5,2 s 2,0,0,0,-s 6,-s 6, s 5,0,0,0,0,-s 6, s 4,0,0,0,0,0,0$

$g_{6,81} \quad 0, s 6,-s 4,-\epsilon s 6, s 2, s 1,-s 6,0, s 5, s 2,0, s 3,0,0,0,-s 4,-s 5,0,0,0,0,0,-s 6, s 4,0,0,0, s 6,0, s 5,0,0,0,0,0,0$

$g_{6,82}-2 \delta s 6,-s 4, s 2,0,0, s 1,0,-(\delta+a) s 6,0,0,0, s 3,0,0,-(\delta+b) s 6,0,0, s 5,0,0,0,-(\delta+a) s 6,0,-s 4,0,0,0,0,(b-\delta) s 6, s 2,0,0,0,0,0,0$

$g_{6,83}-2 \delta s 6, s 5,-s 4,0,0, s 1,0,-(\delta+a) s 6, s 6,0,0,-s 3,0,0,-(\delta+a) s 6,0,0, s 2,0,0,0,(a-\delta) s 6,-s 6,-s 4,0,0,0,0,(a-\delta) s 6, s 5,0,0,0,0,0,0$

$g_{6,84} \quad 0, s 5,0, s 4, s 1,0,0, s 5,0,-s 3,0,0,0,0,-s 6,0,0,0,-s 6,-s 2,0,0,0,0,0$

$g_{6,85}-2 s 6,-s 4,-s 5,-s 2,-s 3,-2 s 1,0,-(a+1) s 6,0,0,0,-s 2,0,0,-s 6,0, s 6,-s 3,0,0,0,(a-1) s 6,0, s 4,0,0,0,0,-s 6, s 5,0,0,0,0,0,0$ 
Citation: Thompson G, Hettiarachchi C, Jones N, Shabanskaya A (2014) Representations of Six-dimensional Mubarakazyanov Lie algebras. J Generalized Lie Theory Appl 8: 211. doi: 10.4172/1736-4337.1000211

Page 7 of 10

\begin{tabular}{|l|l|}
\hline$g_{6,87}$ & $-2 s 6, s 2, s 2-s 5, s 2+s 3-s 5, s 2+s 3-s 4-s 5,2 s 1,0,-s 6,-s 6,0,0, s 4,0,0,-s 6,-s 6,0, s 3,0,0,0,-s 6,-s 6, s 5,0,0,0,0,-s 6, s 2,0,0,0,0,0,0$ \\
\hline$g_{6,88}$ & $-2 a s 6,-s 2,-s 3,0,0,-s 1,0,-(\alpha+a) s 6, b s 6,0,0, s 4,0,-b s 6,-(\alpha+a) s 6,0,0, s 5,0,0,0,-(\alpha+a) s 6, b s 6,0,0,0,0,-b s 6,-(\alpha+a) s 6,0,0,0,0,0,0,0 g 6,88$ \\
\hline$g_{6,89}$ & $-2 a s 6,-s 2,-s 3,0,0,-s 1,0,-(\alpha+a) s 6, b s 6,0,0, s 4,0,-b s 6,-(\alpha+a) s 6,0,0, s 5,0,0,0,-(\alpha+a) s 6, b s 6,0,0,0,0,-b s 6,-(\alpha+a) s 6,0,0,0,0,0,0,0$ \\
\hline$g_{6,90}$ & $-2 \delta s 6,0,-2 s 2,-s 5,-s 3,-2 s 1,0,-(\delta+a) s 6,0,0,0,-2 s 2,0,0,-(\delta+a) s 6,0,0, s 4,0,0,0,-\delta s 6,-b s 6,-s 3,0,0,0, b s 6,-\delta s 6, s 5,0,0,0,0,0,0$ \\
\hline$g_{6,92}$ & $-2 \delta s 6, s 5,-s 3,-s 4, s 2,-2 s 1,0,-\delta s 6, a s 6,0,0,-s 3,0,-a s 6,-\delta s 6,0,0,-s 5,0,0,0,-\delta s 6,-s 6,-s 2,0,0,0,0,-\delta s 6,-s 4,0,0,0,0,0,0$ \\
\hline$g_{6,93}$ & $-2 \delta s 6,-s 2,-s 5, s 4,-s 3,-2 s 1,0,-\delta s 6, a s 6,-s 6,0, s 4,0,-a s 6,-\delta s 6,0,-s 6,-s 3,0,0,0,-\delta s 6, a s 6, s 2,0,0,0,-a s 6,-\delta s 6, s 5,0,0,0,0,0,0$ \\
\hline$g_{6,94}$ & $-(a+2) s 6, s 5,0, s 3, s 1,0,-(a+1) s 6, s 5,0,-s 2,0,0,-a s 6,0, s 3,0,0,0,-2 s 6, s 4,0,0,0,0,0$ \\
\hline$g_{6,95}$ & $-2 s 6,-s 5, s 4, s 3-s 6,-s 2,-2 s 1,0,-s 6,-s 5,0,-s 3,-s 2,0,0,0,0,0,0,0,0,0,-2 s 6,0,-2 s 4,0,0,0,0,-s 6, s 5,0,0,0,0,0,0$ \\
\hline$g_{6,96}$ & $-3 s 6,-s 5,-s 4, s 3-s 5, s 2-1$ \\
\hline$g_{6,97}$ & $2 s 4,3 s 1,0,-2 s 6,-s 5,-s 6, s 3,2 s 2,0,0,-s 6,0,-s 6, s 3,0,0,0,-2 s 6,0,2 s 4,0,0,0,0,-s 6, s 5,0,0,0,0,0,0$ \\
\hline$g_{6,98}$ & $-4 s 6,-s 5, s 3,1$ \\
\hline$g_{6,99}$ & $-s 3-s 4, s 2,4 s 1,0,-3 s 6,0,-s 5, s 3,3 s 2,0,0,-2 s 6,-s 6,0,2 s 4,0,0,0,-2 s 6,0,2 s 3,0,0,0,0,-s 6, s 5,0,0,0,0,0,0$ \\
\hline
\end{tabular}

Table 10: Matrix Representations for Mubarakzyanov Algebras.

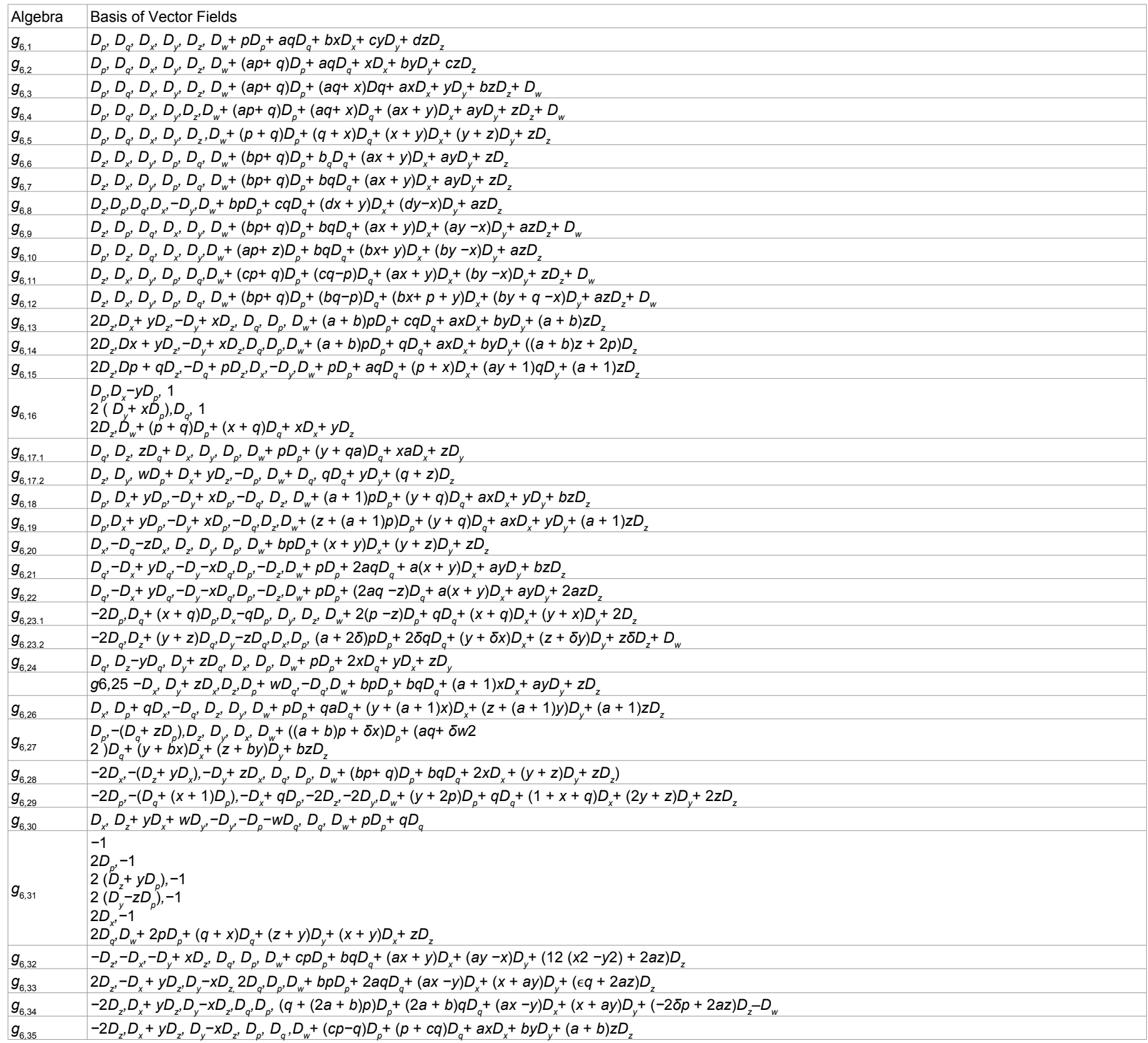


Citation: Thompson G, Hettiarachchi C, Jones N, Shabanskaya A (2014) Representations of Six-dimensional Mubarakazyanov Lie algebras. J Generalized Lie Theory Appl 8: 211. doi: 10.4172/1736-4337.1000211

Page 8 of 10

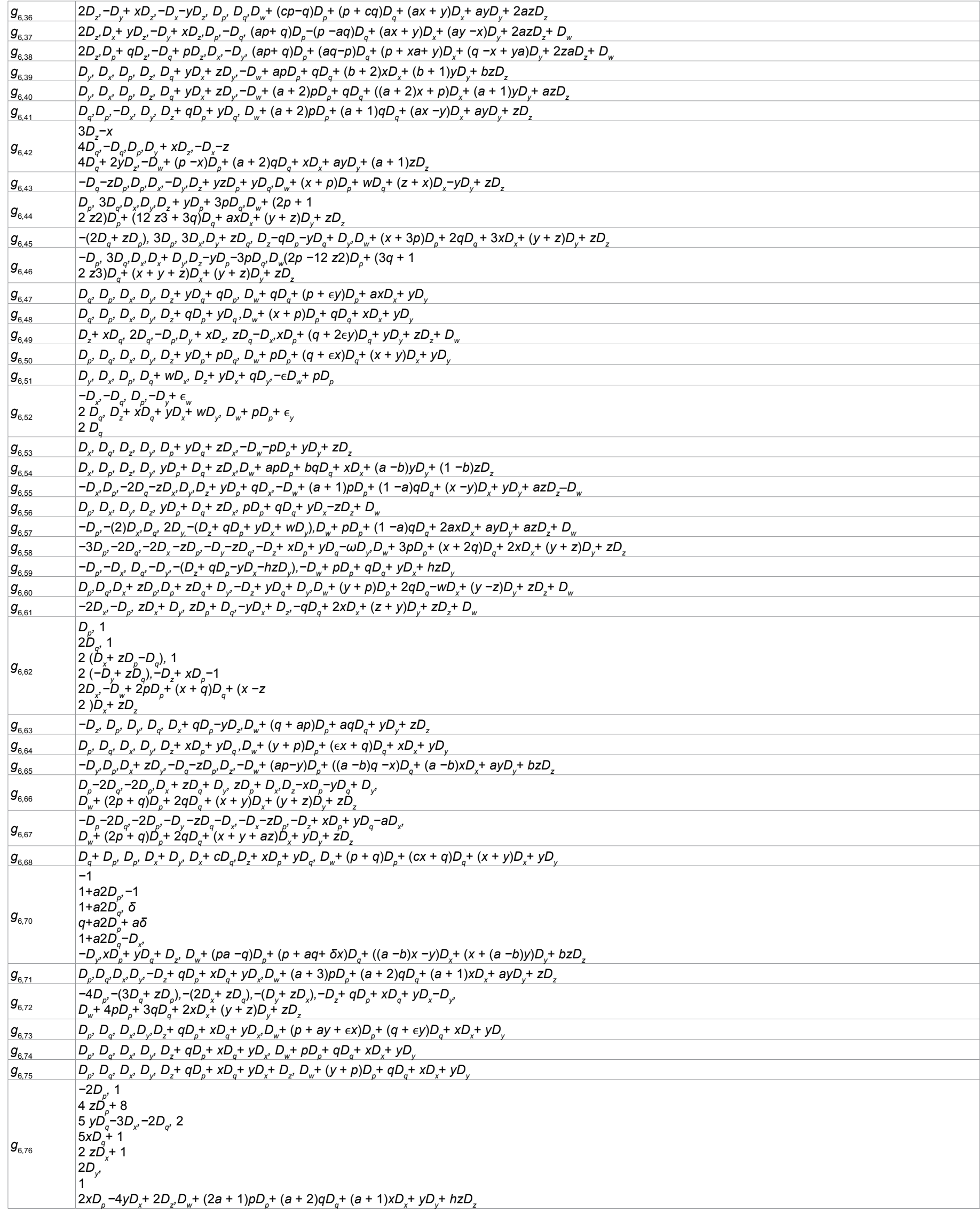


Citation: Thompson G, Hettiarachchi C, Jones N, Shabanskaya A (2014) Representations of Six-dimensional Mubarakazyanov Lie algebras. J Generalized Lie Theory Appl 8: 211. doi: 10.4172/1736-4337.1000211

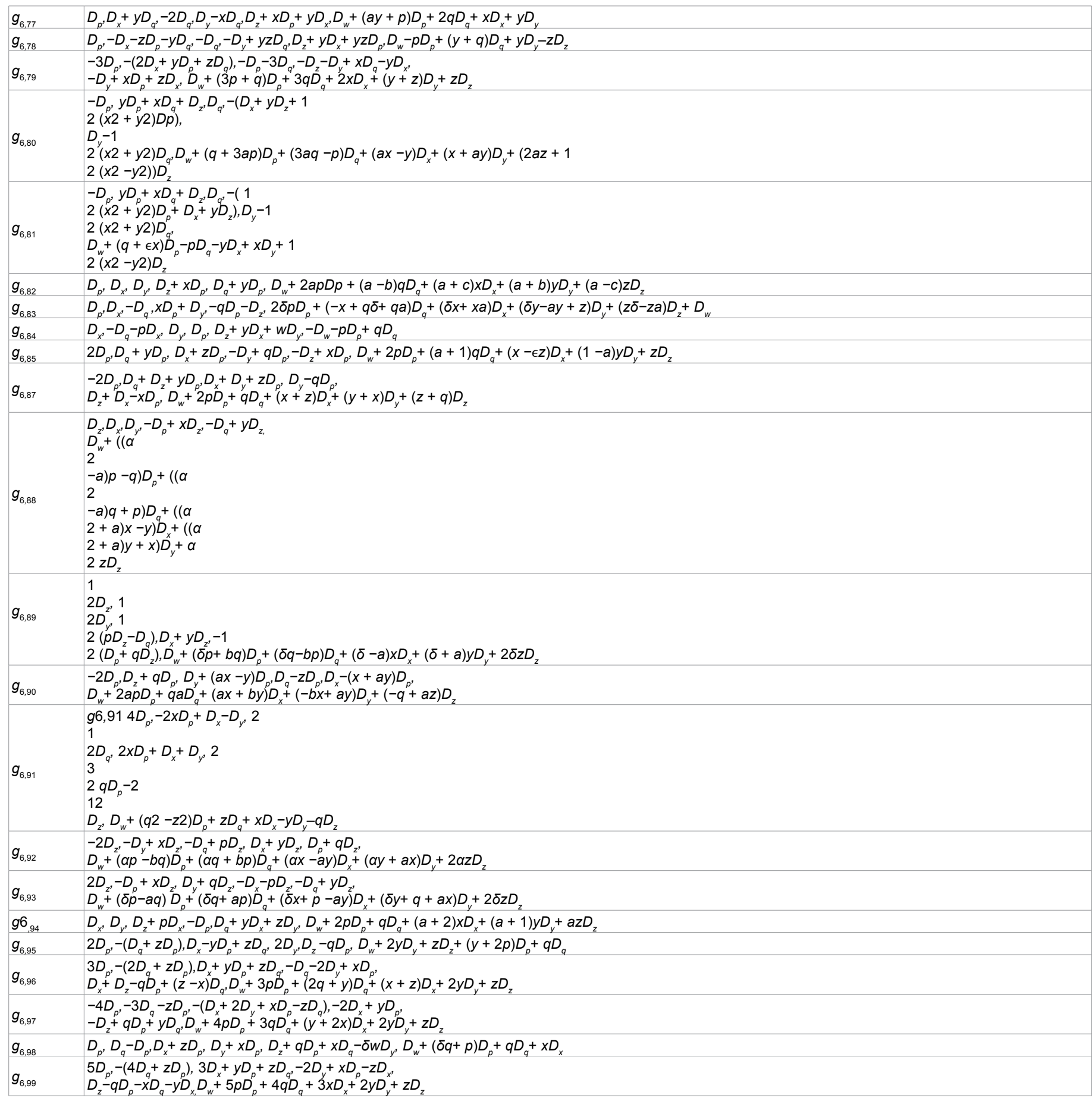

Table 11: Vector Field Representations for Mubarakzyanov Algebras.

- Algebras that are marked with ${ }^{\dagger}$ are suspensions in the sense of Table 3 in Shabanskaya and Thompson [2]

- Algebras that are marked with ' supersede a Mubarakzyanov algebra that is problematic (Tables 10 and 11).

\section{References}

1. Mubarakzyanov GM (1963) Classification of Solvable Lie Algebras in dimension six with one non-nilpotent basis element. Izv. Vysshikh Uchebn. Zavedneii Mat 4: 104-116.
2. Shabanskaya A, Thompson G (2013) Six-dimensional Lie algebras with a fivedimensional nilradical. Journal of Lie Theory 23: 313-355.

3. Burde D (1996) Left Invariant Affine Structures on Reductive Lie Groups. Journal of Algebra 181: 884-902.

4. Burde D (1998) On a refinement of Ado's theorem, 70, Archiv der Mathematik 125-132.

5. Ghanam R, Strugar I, Thompson G (2005) Matrix representations for Low Dimensional Lie algebras. Extractae Mathematicae 20: 151-184. 
Citation: Thompson G, Hettiarachchi C, Jones N, Shabanskaya A (2014) Representations of Six-dimensional Mubarakazyanov Lie algebras. J Generalized Lie Theory Appl 8: 211. doi: 10.4172/1736-4337.1000211

6. Ghanam R, Thompson G, Tonon S (2006) Representations for six-dimensional nilpotent Lie algebras. Hadronic J 3: 299-317.

7. 7.Rawashdeh M, Thompson G (2006) The inverse problem for six-dimensional codimension two nilradical Lie algebras. J Math Phys 47: 112901.

8. Morozov VV (1958) Classification of Nilpotent Lie Algebras in dimension six Izv. Vysshikh Uchebn 4: 161-171.

9. Mubarakzyanov GM (1963) Classification of real Lie Algebras in dimension five. Izv. Vysshikh Uchebn. Zavedneii Mat 34: 99-106.

10. Basarab HP, Lahno V, Zhdanov R (2001) The structure of Lie algebras and the classification problem for partial differential equations. Acta Math Appl 69: 43-94.
11. Campoamor RS (2005) Some remarks concerning the invariants of rank one solvable real Lie algebras. Alg Colloquium12: 497-518.

12. Patera J, Sharp RT, Winternitz P, Zassenhaus $H$ (1976) Invariants of real low dimension Lie algebras. J Math Phys 17: 986-994.

13. Turkowski P (1990) Solvable Lie Algebras of dimension six. J Math Phys 31 : 1344-1350.

14. Turkowski $P$ (1994) Literature on the structure of low dimensional nonsemisimple Lie algebras and its applications to Cosmology. Acta Cosmologica 20: 147-153. 